\title{
Hypnerotomachia Poliphili and Its Afterlife
}

\author{
Guo Quanzhao ${ }^{1, \text { a }}$
}

\author{
${ }^{1}$ School of Translation Studies, Shandong University (Weihai), Weihai, China \\ alouzhangguo@163.com
}

\begin{abstract}
Hypnerotomachia Poliphili has been applauded as the most beautifully printed book in the Western Renaissance and the most difficult one to understand. It was once cloaked in mysteries and enigmas, and its influence rippled through literature, art and architecture. It is a love romance of the Italian Renaissance, an encyclopedia in the guise of a novel, and probably the world's longest book about people dreaming. This paper briefly summarizes its dissemination, influence and the relevant critical views, focusing on its plot, structure, language, rhetoric and other literary characteristics, while making an investigation of its literary genealogy and contemporary inheritance. By analyzing the re-appropriation of the literary formula of Hypnerotomachia Poliphili by Umberto Eco in his novel The Mysterious Flame of Queen Loana, it is argued that excellent worksand brilliant artists can revive our history, tradition and past life, by employing the so-called "Aby Warburg's Approach", and thus responding to the issues and concerns of the present age.
\end{abstract}

Keywords: Hypnerotomachia Poliphili, Renaissance, Umberto Eco, The Mysterious Flame of Queen Loana, Aby Warburg's Approach

\section{《寻爱绮梦》及其死后生命}

\section{郭全照 ${ }^{1, a}$}

1 山东大学 (威海) 翻译学院, 威海, 山东, 中国

alouzhangguo@163.com

\section{摘要}

《波利菲洛寻爱绮梦》被誉为西方文艺复兴时期印刷最精美的书，也是最难懂的书。它一度披着种种谜团和神 秘色彩, 影响波及文学、艺术和建筑。它是意大利文艺复兴时期的一本浪漫爱情小说, 也是一部伪装成小说模 样的百科全书, 可能也是世界上最长的一部描写人做梦的书。本文简要总结了其传播、影响和相关批评观点, 聚焦于《寻爱绮梦》的情节、结构、语言、修辞等文学特征, 并考察了其文学谱系和当代传承。通过分析艾柯 小说《罗安娜女王的神秘火焰》对《寻爱绮梦》文学程式的重新挪用，论证优秀作品和杰出艺术家的 “瓦尔堡 方法”可以复活历史、传统和旧的生命，回应现时代的问题和关怀。

关键词: 《寻爱绮梦》, 文艺复兴，艾柯，《罗安娜女王的神秘火焰》，瓦尔堡方法

\section{1.文艺复兴奇书}

爱书人和藏书家都应该知道珍稀的摇篮本。所谓 “摇篮本”（incunabula）, 专指西方印刷史初期, 在 1450-1500 年间, 使用活字印刷术印制出版的书籍和 小册子。那是西方书籍由手抄本进入印刷时代的滥解。 1450-1455 年印制完成的古腾堡圣经（Gutenberg Bible), 也称“42 行本圣经”, 西方活字印刷生产的第 一本书, 为摇篮本时代的开始; 而 1499 年在威尼斯
出版的《寻爱绮梦》 (Hypnerotomachia Poliphili, 直 译为《波利菲洛寻爱绮梦》, 简称《寻爱绮梦》, 下同), 则为摇篮本时代的结束。这两部书都有堪称完美的编 辑排版技艺, 图文结合, 呈现精良, 被公认为西方印 刷史上的巅峰之作，《寻爱绮梦》更被冠以“文艺复兴 时代最美的书”的荣名。

西书收藏在中国一直处于“小众”地位, 摇篮本的 收藏更是“小众中的小众”。据最新统计，中国包括大 
陆和台湾两地共有仅 54 种 56 册摇篮本 (Bookman57)。 2019 年 2 月, 在台北第二届春风似友珍本古籍拍卖 会上, 意大利摇篮本《寻爱绮梦》以 450 万新台币 (约 合人民币 98 万）成交，创下亚洲拍场西文古籍第一 高。《寻爱绮梦》由此正式走入汉语世界—但仅仅 是收藏界, 比如书籍史研究者刘兴华、台湾作家钟芳 玲都从出版、收藏、书籍史、艺术史角度写过介绍文 章。此外和此前是建筑界, 在 2011 年, 由南京大学 建筑学院与南京大学人文社会科学高级研究院主办 的年刊《建筑文化研究》第三辑中收录了一个关于《寻 爱绮梦》的文章专辑, 主要是国外研究者 (如安东 尼·布朗特[Anthony Blunt]、贡布里希[E. H. Gombrich] 罗斯玛丽. 特里普[Rosemary Trippe]、罗斯维塔.施特 林 [RoswithaStewering]、阿尔伯托. 佩雷兹-戈麦兹 [Alberto Pérez-Gómez]、里安娜. 莱夫维尔 [Liane Lefaivre]）相关文章的译文, 涉及《寻爱绮梦》的传 播、影响、作者、建筑插图及其文学批评, 书前有南 京大学建筑学院胡恒教授的一篇综述, 梳理了 500 年 来《寻爱绮梦》的版本、译本情况, 但这些文章在中 国国内学术界没有引起什么反响。而中国国内文学研 究界对这部“文艺复兴奇书”的关注尚付之成如。

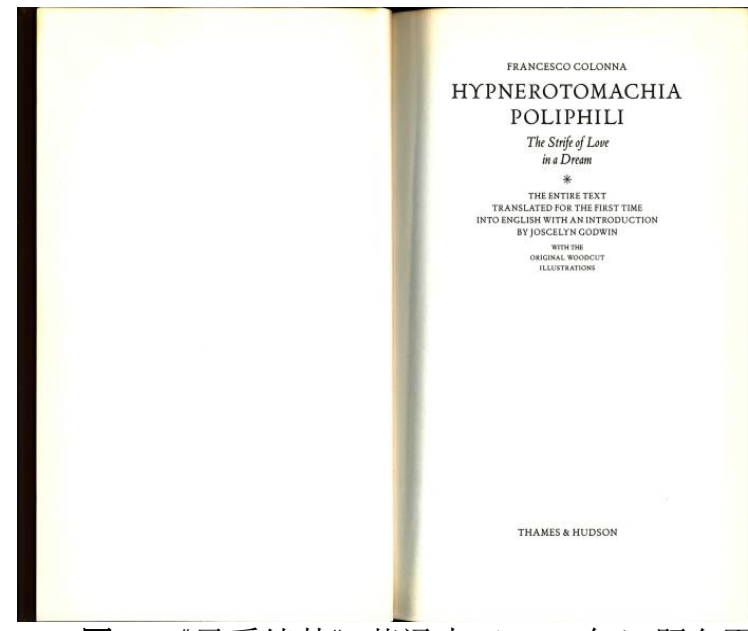

图 1 《寻爱绮梦》英译本（1999 年）题名页

“奇书”之“奇”，首在作者身份成谜。初版本的题 名页上并没有作者署名, 传统上虽归之于弗朗切斯科 •科隆纳（Francesco Colonna, 1433-1527)（因为将每 个章节的起首大写字母串联起来, 包含“弗朗切斯科・ 科隆纳”这个名字), 但也有其他可能, 如意大利建筑 大师阿尔伯蒂 (Leon Battista Alberti, 1404-1472)、文 艺复兴佛罗伦萨统治者洛伦佐·德·美第奇 (Lorenzo de' Medici，1449-1492）及此书印刷商阿尔杜斯（Aldus Pius Manutius, 1449/1452-1515) (Bookman 63; Wowo and Heng 178-191)。其图文结合的不寻常方式（全书 从头到尾配有 172 幅木刻插图), 如杯型文、倒锥文、 两图夹文、跨页连幅等新奇的排版超越了时代, 具有 20 世纪先锋派印刷品的特质 (Wowo and Heng 8)。通 过结合拉丁语、希腊语和意大利语方言, 《寻爱绮梦》 的语言晦涩难懂却自成一派: 词汇大多由拉丁词加上 意大利语词尾构成, 小部分有希腊语词源, 另有少量 铭文中出现的希伯来语、阿拉伯语、迦勒底语(Godwin,
“Introduction" IX-X in Colonna)。其在语言上的大胆 实验, 与乔伊斯（James Joyce, 1882-1941）等现代派 作家相比也不遑多让。其在知识构成上是百科全书式 的, 涵盖历史、地理、建筑、植物、动物、矿物、数 学、技术工艺等多门学科, 其中建筑博物学是主体, 此外还涉及神话学、占星术、炼金术、象形文字、古 代仪式等方面的知识（Wowo and Heng 3,14）。其在 后世的影响遍及园林建筑、绘画和文学, 研究者的兴 趣则集中于作者的身份、版本的流传、插图的图像和 文学渊源、图像和文本的关系。

它是一个爱情罗曼司、梦幻文学和色情描写的混 合体。它继承、改造和发展了但丁 (Dante)、彼特拉 克 (Petrarch) 和薄伽丘 (Boccaccio) 的方言文学传 统，《玫瑰传奇》(The Romance of the Rose) 的梦幻文 学传统, 奥维德 (Ovid)、阿普列乌斯 (Apuleius)、 老普林尼 (Pliny the Elder) 的神话与拉丁词源学传统, 及建筑书写传统 (Godwin, “Introduction” IX-XI in Colonna)。如果说它在语言上的独创性在于将意大利 语句法和拉丁语词汇相结合, 那么它在文学上的独创 性就在于利用既有程式, 以古典词汇和大量幻想的结 合、博学与情爱的结合, 建造了一个多神论的异教世 界，主体在其中恢复了智性和感性、自然和文明、爱 欲和神性之间的奇妙联结。

\section{2. 《寻爱绮梦》的奇幻旅程}

题名中的 Hypnerotomachia 混合了三个希腊词语, hypos 意为“睡觉” (sleep), eros 意为“爱” (love), mache 意为“争斗”、“纷争” (strife)。所以, 1999 年 出版的第一个英文全译本的副标题就是: “梦中爱的 纷争” (the strife of love in a dream)。Poliphilo 这个名 字的意思是“爱波莉亚”( lover of Polia), 但也是“爱 许多事物"( lover of many)(Godwin, “Introduction" VII in Colonna)。小说的副文本（paratext）也非常丰富。 除了插图，正文之前，首先是出版商“维罗纳的列奥 纳多・格拉索”（Leonardo Crasso of Verona）给赞助人 “乌尔比诺公爵圭多”(Guido, Duke of Urbino)的献词, 声称本书是他“偶然获得”、自费印刷的。这个格拉索 提到了小说的三个特点: 涉及广泛知识、多种语言、 包含许多插图 (illustrations) 和图像 (images)。格拉 索并以“读者” (reader; gentle reader) 为呼语, 介绍小 说梗概, 提请读者注意其中的情色内容 (the new erotic guest), 重点是对这部书的称颂。而在波利菲洛开始 第一人称叙述之前, 又有一份他给波莉亚的献词, 重 点是对波莉亚的称颂和表白 (Colonna 2-8)。小说正 文没有像现代小说那样标明章节, 而是每到情景或情 节转换处轩以大写字母写就的“波利菲洛（或波莉亚） 如何如何” (小说后半部分故事和叙述主体换成了以 波莉亚为主) 的一小段话 (一般由一、两个句子组成) 过渡，起到了划分的作用。 


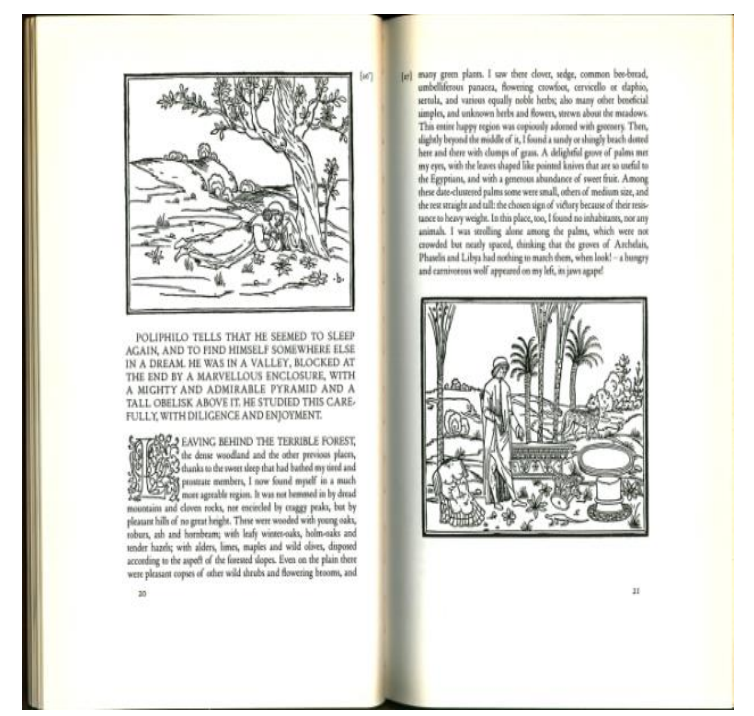

图 2 《寻爱绮梦》英译本（1999 年）插图: 波 利菲洛树下入梦

书分为两卷, 38 个章节。故事一开始, 波利菲 洛因想念波莉亚而一夜无眠, 终于在黎明时分缓缓进 入梦乡。梦中的波利菲洛来到一片幽暗森林, 心中怀 着恐惧胡乱游走, 被歌声埽引到一棵老像树下, 再次 陷入睡眠。在第二层梦境, 他游荡于纪念碑、废墟、 花园、宫殿、庙宇之间, 并加以仔细观察和详细描述。 他先是发现自己置身山谷之中, 远处山丘相接处有一 个头顶方尖碑的巨大金字塔。进入一个宏伟大门之后, 在古代柱廊尽头的门槛处窝出一条恶龙。波利菲洛慌 忙逃进门槛后面的黑暗之中, “我在这黑暗洞穴的腹 内, 甬道弯弯曲曲, 看不清周围, 我身处的恐惧和险 情, 比墨丘利 (Mercury)被变成朱鹭、狄安娜 (Diana) 被变成寒鸦、潘神 (Pan) 生就半人半羊、阿波罗 (Apollo) 在色雷斯遭到放逐还要为甚, 也比俄狄浦 斯王 (Oedipus)、古列王 (Cyrus)、克里萨斯王 (Croesus) 或珀尔修斯 (Perseus) 所处的为甚” (Colonna 63)。 他经常拿各种神话人物和神话故事作为类比, 这也是 小说在修辞上的一个显著特色。他逃到金字塔内部, 从另一侧的出口进入了一个美好的国度, 统治者是智 慧、慷慨、仁善的埃勒泰瑞里达 (Eleuterylida) 女王。 他遇到了女王的五个仙女 (nymphs) 侍从, 受邀跟她 们一起沐浴 (在沐浴谈话时, 一个仙女向波利菲洛解 释了他名字的含义, 即“泛爱”[much loving]和“波莉亚 的情人”[friend of Polia]), 并参加在女王宫殿中举行 的盛大宴会。然后, 女王派另两位仙女引导他, 穿过 三座花园, 来到三座带有铭文的黄铜门前, 他必须选 择其一。他打开了中间那扇门, 见到了手持火炬的波 莉亚。奇怪的是, 波莉亚叫得出他的名字, 却似乎并 不认识他。这也是两人的第一次相遇, 直到全书约三 分之一的地方。此处, 波利菲洛细致描写了波莉亚的 外貌、衣服和装饰, 毫不文惜赞美之词。她的美带给 波利菲洛的震撼远大于美与爱之神维纳斯 (Venus) 给战神玛尔斯 (Mars)、英俊牧羊人阿多尼斯 (Adonis) 给维纳斯、美少年伽倪墨得斯（Ganymede）给朱庇 特 (Jupiter)、美少女普绪克 (Psyche)给丘比特 (Cupid)
的震撼 (Colonna 143)。两人相偕前行, 观赏主神朱 庇特的凯旋队伍, 见到了众多仙女, 领略了美妙的仙 境。然后, 他们步入维纳斯的神庙, 意识到了彼此的 爱。两人离开神庙后, 来到海边, 等待时, 波利菲洛 独自探访了附近的古城。返回海边后, 他跟波莉亚一 起登上丘比特的小船, 到达了塞西拉岛 (the Isle of Cytherea）——圆形的爱之岛。在岛的中心, 维纳斯 喷泉（the Fountain of Venus）就是他们此行的终点。 在那里, 维纳斯女神送给他俩紫水晶戒指和仙女伴护, 丘比特用箭射穿了他俩的心脏, 维纳斯为他俩举行了 结合仪式。当寻求在维纳斯怀里安睡的玛尔斯到达后, 波利菲洛、波莉亚跟他们的仙女伴护离开圣泉, 来到 阿多尼斯之墓, 在这里, 仙女们讲述了维纳斯对逝去 的情人阿多尼斯的怀念 (维纳斯每年都会带儿子丘比 特前来举行神秘仪式, 纪念阿多尼斯)。最后, 仙女 请波莉亚讲述她的家族世系和爱情经历。这就是第一 卷的主要内容, 波利菲洛的梦中漫游。

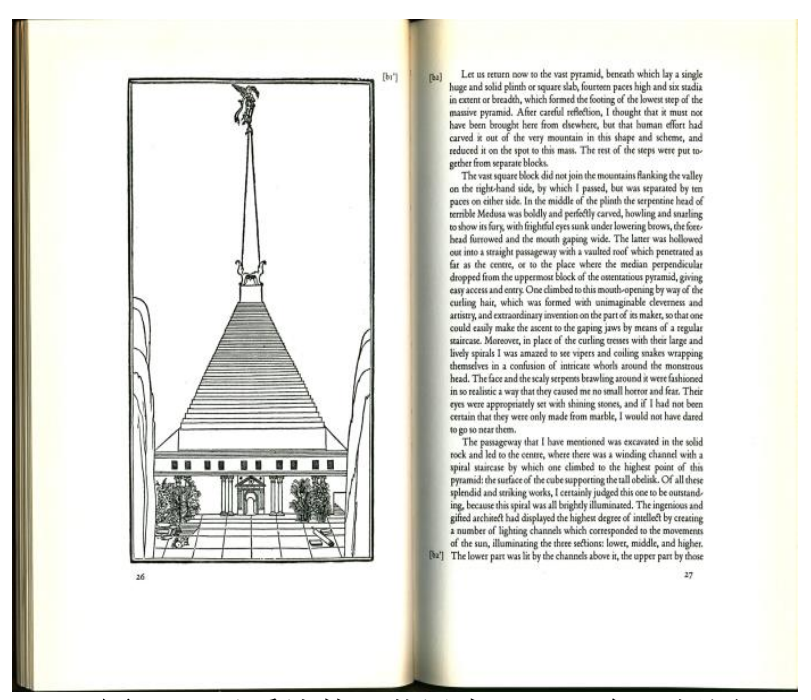

图 3 《寻爱绮梦》英译本（1999 年）插图: 金字塔

第二卷相比之下, 简短了很多, 少了关于建筑和 艺术品的描绘, 以波莉亚为主要叙述者, 讲述二人之 间曲折的爱情经历。波莉亚首先追溯了自己的家世, 她的祖先是古罗马执政官, 设立并保护着特雷维索城

(Treviso), 她还提到自己出生于 1462 年。有一天, 波莉亚在窗前梳理自己的一头金发, 波利菲洛偶然路 过, 对她一见倾心。自此, 波利菲洛日日来她窗下瞻 望、弹琴唱诗, 波莉亚却对他无动于衷。不料城内爆 发瘟疫, 大量人口死亡, 人人自危, 都去城外躲避。 人们猜测, 瘟疫传自埃及, 因为他们认为, 浑浊的尼 罗河水里满是动物尸体而恶臭不堪, 空气也受到污染, 继而南风把疫疠带到了特雷维索。波莉亚也身受其害, 腹股处罹患肿瘤, 众人避之唯恐不及, 唯有家人一样 的保姆对她不离不弃。波莉亚向狄安娜女神祈求救护, 不久竟神奇地自愈了。她愈加虔诚地信奉狄安娜, 参 加狄安娜神庙的祝圣礼 (consecration) 时, 被前来观 礼的波利菲洛撞见。波莉亚忠于对狄安娜的守贞誓言, 
面纱罩面，对波利菲洛避而不见。焦虑、绝望的波利 菲洛抓住波莉亚在庙内独自祈祷的时机，现身表达爱 意。看出波莉亚对自己无意也无情, 经历了长时间单 相思的痛苦和折磨，波利菲洛晕死在了波莉亚面前。 而波莉亚竟然冷酷地把波利菲洛的尸体拖到神庙一 角, 然后想偷偷溜回家中。路上, 波莉亚被一阵旋风 刮到了一片险恶难行的树林, 看到两个少女被一个带 翼小孩虐杀，遭狗、狼、狮、鹰分尸，她顿时悚惧涕 泣、心惊胆寒。失魂落魄的波莉亚返回家中，做了一 个哥梦, 梦到两个刽子手找上门来。在保姆的劝导之 下, 她意识到自己触怒了爱神, 痛悔于自己的冥顽不 灵。思过后, 波莉亚心中升起了对波利菲洛的爱欲, 她转天回到狄安娜神庙, 抱住波利菲洛的尸身痛哭, 波利菲洛就在她的拥抱中活转过来。两人被狄安娜神 庙的女祭司们赶了出去。波莉亚转投维纳斯, 誓言忠 于爱神和波利菲洛。在维纳斯神庙, 两人互诉衷肠, 波莉亚向波利菲洛大胆示爱, 维纳斯赞许二人真情结 合, 但要听波利菲洛讲他爱上波莉亚的完整、详细过 程。于是, 波利菲洛又讲述了一遍他如何在狄安娜神 庙见到波莉亚、给波莉亚写了三封情书、在波莉亚独 自祈祷时现身相见、在波莉亚身边死去又在波莉亚怀 中复活的整个经过。波利菲洛的灵魂曾在他死后升到 天上, 向维纳斯诉说自己的苦恋之难。维纳斯在波利 菲洛的灵魂的恳求之下, 命令丘比特用箭去射波莉亚, 这样一来, 波莉亚就会爱上这个为她而死的男子。最 后, 天界和人间诸事终于让波莉亚爱上了波利菲洛。 仙女们听完波莉亚的叙述, 心满意足地离开了, 独留 两人深情倾诉并热吻。在难以想象的喜悦当中, 波莉 亚轻烟般消失，波利菲洛的梦也醒了。

《寻爱绮梦》在结构上并不是一个单线和单纯的 叙事, 而是一系列故事、梦幻、演说和信件互相嵌套 的迷宫。两卷书的叙述视角和风格也不一样。第一卷 完全依循波利菲洛的视角, 第二卷则以波莉亚的声音 为主。波利菲洛的叙述富有唯美主义的沉思意味, 波 莉亚的叙述则更显世俗化, 有的故事近于薄伽丘《十 日谈》的粗俗和幽默, 如波莉亚关于自己遭遇两个㓣 子手的哥梦 (Colonna 405-407)、她的保姆所讲的一 个年轻女子嫁给老汉陷入悲惨婚姻而后悔自杀的故 事（Colonna 412-415)。第一卷的背景是无时间性的 想象之域, 第二卷则设定在真实的意大利东北部的特 雷维索城。第一卷的高潮出现在位于塞西拉岛中央的 维纳斯喷泉, 波利菲洛和波莉亚在女神的见证和祝福 下“结婚”。第二卷的高潮在天界, 波利菲洛的灵魂向 维纳斯求助。故事刚开始不久, 波利菲洛就进入了梦 中之梦 (第二层梦境)。在波莉亚角度的叙述中, 波 莉亚也做过梦。从整个故事是波利菲洛的一个梦这个 叙事框架来看, 波莉亚的梦就是第三层梦境。而第二 卷波利菲洛叙述中的他的灵魂历险又是处于波莉亚 视角之中的, 因而应是第四层梦境。小说结尾, 波利 菲洛独自醒来, 波莉亚的幻影也随之消散。事实上, 梦中发生的一切都是波利菲洛的幻想, 包括波莉亚口 述的波利菲洛的经历。这是一部情爱之书, 波利菲洛 为了未曾见过就爱上的波莉亚魂牵梦绕、出生入死,
波利菲洛的保护神是爱神维纳斯和丘比特, 波莉亚一 开始崇拜狄安娜, 后来也转向维纳斯和丘比特, 还有, 行文中有很多明显的性暗示甚至明示 (例如, 描写建 筑的语言感性而色情，像“极乐”、“心荡神迷”、“激情 难抑”等词汇充斥在建筑描述之中)。这又是一部博爱 之书, 波利菲洛喜爱建筑、园林、碑刻、雕塑、浮雕、 金银匠艺、织物、音乐、庆典、仪式，他对衣饰鞋子 和对精美喷泉、象征性方尖碑都同样迷恋。如果从柏 拉图主义的定义来看, eros 就意味着对各种美的事物 的热爱。

波利菲洛喜欢各种珍宝和密集、象征性的内部装 饰, 这种趣味预示了托斯卡纳大公、弗朗西斯科一世 (Francesco de' Medici, 1541-1587) 的普拉托利欧

(Pratolino) 庄园和佛罗伦萨维奇奥宫（Palazzo Vecchio), 提洛尔大公费迪南二世 (Ferdinand II, 1529-1595) 的阿姆布拉斯宫 (Schloss Ambras), 神 圣罗马帝国皇帝鲁道夫二世（Rudolf II, 1552-1612） 的布拉格城堡（Pražskýhrad）, 巴伐利亚国王路德维 希二世（Ludwig II, 1845-1886）的新天鹅堡（Schloss Neuschwanstein)。它们都布满了来自自然和艺术的奇 珍异宝、古怪事物、园林建筑 (如花园、喷泉、雕塑、 岩洞等), 处于梦幻和现实的交界处。17、18 世纪直 到今天, 凡尔赛宫 (Palace of Versailles) 的园艺设计 也受到《寻爱绮梦》插图的影响 (Godwin, “Introduction" XVI in Colonna; Bookman 89, 96; Wowo and Heng $4-5$ )。

《寻爱绮梦》的图像元素被后世的许多画家吸收 和转化, 如意大利文艺复兴时期威尼斯画派画家乔尔 乔内 (Giorgione, 1477/1478-1510) 的《沉睡的维纳斯》、 《暴风雨》, 提香 (Titian, 1488/1490-1576) 的 《乌尔 比诺的维纳斯》、《天上的爱与人间的爱》, 法国洛可 可代表画家华托 (Antoine Watteau, 1684-1721) 的《舟 发西苔岛》等 (Bookman 78-96; Wowo and Heng 4, 36-43)。《寻爱绮梦》也含有一种世纪末颓废艺术 (fin-de-siécle) 的气息, 回响在于斯曼 (Joris-Karl Huysmans, 1843-1907)、王尔德 (Oscar Wilde, 1854-1900)、比亚兹莱 (Aubrey Beardsley, 1872-1898) 等人的作品里。由比亚兹莱的黑白插画及其情色小说 《维纳斯与唐豪瑟》(Venus and Tannhäuser), 可见出 他知道《寻爱绮梦》并热爱它。于斯曼的小说《逆天》

(À Rebours) 以对没落贵族德泽森特 (Des Esseintes) 精奢靡费的收藏和装饰爱好的绚丽书写, 赢得了“唯 美主义圣经”的称号, 成为王尔德小说《道连・格雷的 画像》 (The Picture of Dorian Gray) 的模仿先驱 (Godwin, "Introduction" XVI in Colonna)。

\section{3. 《寻爱绮梦》的死后生命}

《寻爱绮梦》中不规则的混杂语言、奇异的怪人 怪事、眼花缭乱而缓慢冗长的激情叙述和百科知识、 密码式的文字和图像、身份难辨的作者, 共同铸就了 “波利菲洛那充满魔力和炼金术的文艺复兴世界”

(Wowo and Heng 146), 就像全书开篇不久的那幅青 
铜马雕像版画: 一匹狂野不羁的带翼奔马, 拱起背部 将那些试图紧贴马背和鬃毛的读者“骑手们”一个个 甩下。尽管公认《寻爱绮梦》是“文艺复兴时期最深 奥难懂的文本之一” (Wowo and Heng 107), 也是“迄 今为止最难读懂的书籍之一” (Wowo and Heng 157), 但它又具有巨大的人文艺术鬼米力和神秘的解谜吸引 力。诞生之初, 因定价昂贵、博学隐晦, 只局限于人 文主义者圈子和贵族阶层, 但随后借着重印和翻译 （1545 年重印本，1546 年、1600 年法译本, 1592 年 英译本等), 其辐射力逐渐蔓延开来。17 世纪的时候, 《寻爱绮梦》还在欧洲广泛流传, 其在哲学、艺术甚 至炼金术领域的重大影响持续到了 18 世纪，直到 19 世纪初, 它还偶现踪影。它的建筑描写 (超过全书三 分之二篇幅) 和建筑插图 (172 幅中的 88 幅) 滋养 了许多建筑师的想象, 成为文艺复兴时期的建筑图册 (Bookman 71), 影响了欧洲建筑设计至少 300 年 (Pérez-Gómez 53-54), 以至当代著名加拿大建筑史 学家阿尔伯托·佩雷兹-戈麦兹 (Alberto Pérez-Gómez) 建议当今大学应将《寻爱绮梦》作为建筑学教材

(Pérez-Gómez 57, 145), 当今西方的建筑理论读本经 常收入《寻爱绮梦》的章节片段 (Wowo and Heng 5)。 欧洲许多画家从它的文字和插图中汲取灵感和造型 源泉, 做出自己的变化和运用。

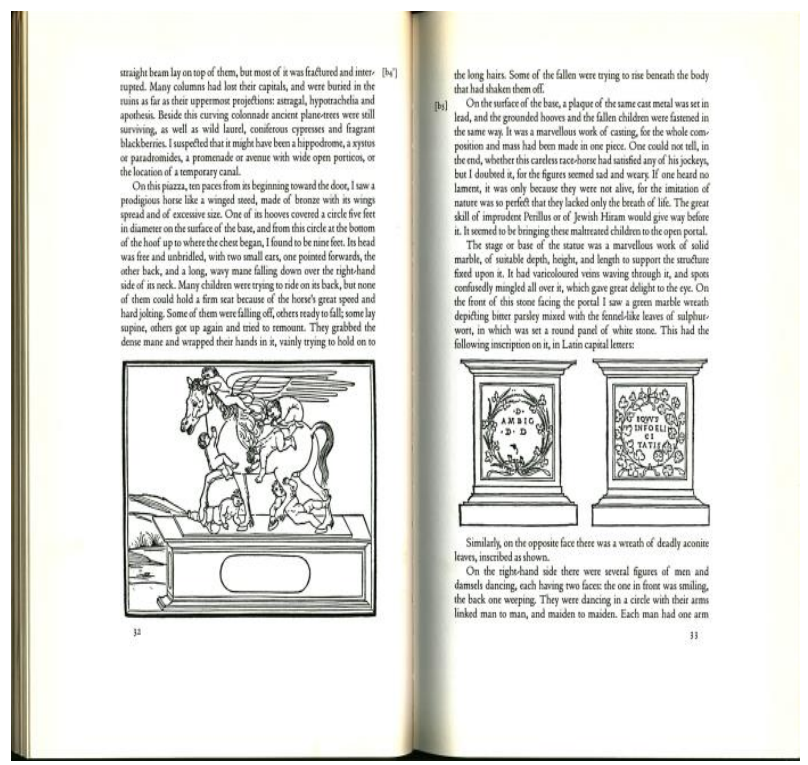

图 4 《寻爱绮梦》英译本（1999 年）插图:

\section{青铜马雕像}

500 多年来, 对于此书的解读五花八门, 艺术史、 文学批评、哲学、史学等理论都曾被用作诠释工具。 16、17 世纪的人认为书中的象形文字和寓意画隐含 了炼金术的秘密 (Wowoand Heng 31); 荣格派学者看 到原型象征, 弗洛伊德式批评家看到恋物情结和性欲 投射（Bookman 67-69; Wowoand Heng 160); 建筑史 学者读出了“我们可以从解释学本体论与现象学中得 到的建筑策略” (Wowo and Heng 155); 史学视角发 现了其中的威尼斯人文主义表现 (关心古代神话和诗 意幻想，不同于 15 世纪佛罗伦萨的理性人文主义，
后者关心的是古代哲学)（Wowoand Heng 44-45）或 是对罗马人文主义精神（简单、真诚、淳朴、自由、 不信教) 的迷恋 (Wowoand Heng 185-190); 哲学视 角找出了新柏拉图主义（爱与美-艺术相关联，色情 和博学相搅拌) 的反映 (Wowo and Heng 160; Pérez-Gómez 54, 110); 在后现代主义视角下, 《寻爱 绮梦》呈现了现代技术性人类的游牧境况 (总在迁徙、 跨越, 而没有一个清晰的目的地)(Pérez-Gómez 139)。

文学史上，拉伯雷、拉封丹作品中的离题延宕、 建筑描写都有《寻爱绮梦》的影子 (Wowo and Heng 28-29); 《寻爱绮梦》以宫廷爱情文学和方言文学 (《玫 瑰传奇》、彼特拉克、薄伽丘等) 作为创作资源, 大 量参考前人文献，但并不简单模仿，而是有所超越， 如对情人外貌的描写, 传统的肖像写法是从头到脚依 次描述情人的典型特征 (金发、黑眼睛、白晳皮肤等), 而《寻爱绮梦》对波莉亚的描写却随意、杂乱，显得 是对所见的即时描述, 因而富于现场感和真实感, 并 使读者对波利菲洛产生情感认同，感受到他无法餈足 的视觉和欲望 (Wowo and Heng 79-82)。《寻爱绮梦》 采纳文学传统作为自己的原始材料, 却做出了自己的 革新, 它在语言的探索和表现上尤其大胆, 其多语言 重组的联想主义风格可视为乔伊斯《芬尼根守灵夜》

（1939 年）的先驱（Wowo and Heng 160）。它在文本 和插图之间建立了多层次的关系 (对照、补充、隐喻, 且插图的功能既可叙述也可描述), 还进行了象形文 字、图画诗这样别出心裁的创造 (Wowo and Heng 82-90)。它用精巧华丽、想象生动的语言, 以神话典 故、各种辞格配合版画, 唤起栩栩如生的图像, 它带 领我们去往的不是作为膜拜和考古对象的古代, 而是 一个梦幻、魔法和想象驰骋的镜中世界，是现实生活 的替代品。如果说，我们今天仍能带着利用或愉悦的 目的来阅读它, 那是因为梦想和爱欲乃是意义之源, 这是我们人性当中过去、现在和将来都不会泯灭的部 分。

今天对《寻爱绮梦》的关注, 多数是把它当成博 物馆里的标本，它对艺术、建筑的激发作用被封存起 来，它具有的迷狂、奔放、迟缓、神秘、漫游等情感 能量和美学灵氛失落了。波利菲洛在他的“梦中梦”世 界经历了死亡和复活, 《寻爱绮梦》在当今世界还能 有第二次生命吗?

德国当代著名作家马丁・莫泽巴赫（Martin Mosebach, 1951- ）认为, “像《寻爱绮梦》这样精雕 细琢、完美无瑕的艺术品仍然能为二十世纪提供素材 和创作的源泉”。他提到的一个例子是由德英法三国 艺术家所创作的《维吉尔》一书, 文图并美, 是“向 阿尔杜斯的旷世杰作的致敬”。但这部《维吉尔》, 文 字内容是对维吉尔田园诗和农事诗的翻译，而不是当 代作家自己的创作（Mosebach 73-78）。2004 年美国 出版过一本悬疑小说《四法则》( The Rule of Four), 其中两个普林斯顿大学的准毕业生探索《寻爱绮梦》 这个“谜团”的过程掺杂着对亲情、友情、爱情的大众 通俗文学表现 (Caldwell and Thomason)。而深入继 
承、发扬和革新《寻爱绮梦》传统的当代文学作品在 哪里呢?

意大利当代著名作家艾柯 (Umberto Eco, 1932-2016）以小说《玫瑰之名》（1980 年）闻世。他 不但是百科全书式的学者、小说家, 也是资深的古书 收藏者。他拥有三十来部印刷初期珍本, 其中就包括 《寻爱绮梦》的初版本（Tonnac 110, 115; Eco 2014: 34 )。他的第五部长篇小说《罗安娜女王的神秘火焰》 (2004 年) 的主人公亚姆柏 (Yambo) 就是以一篇关 于《波利菲洛寻爱绮梦》的论文从大学毕业。亚姆柏 是 1990 年代米兰的一个 60 岁的古书商。小说围绕亚 姆柏的失忆与回忆, 他的两次昏迷, 他清醒时的记忆 搜寻和昏迷中的记忆恢复，追溯二战前后及战争期间 他从童年到青少年的见闻与经历。亚姆柏从看到、读 到和听到的东西中拼凑出一代意大利人的集体记忆, 而他的初恋莉拉 (Lila) 成为通向他个人历史的神秘 入口, 同时, 莉拉也代表了他失去的爱, 一个永远达 不到的“理想”。小说中说《寻爱绮梦》是一部艰深难 懂的 15 世纪意大利古书, 正是因为它, 亚姆柏毕业 后即前往德国研究古书历史, 并在回国后成立了贩卖 古书的事务所 (Eco 2009: 53-54)。《寻爱绮梦》通常 被认为是早期现代印刷史上“最美的书”，整合了文本 与图像 (木刻插图), 艾柯的《罗安娜女王的神秘火 焰》也整合了来自通俗文化、文本的插图: 书中有一 百多幅插图, 来自文本中提到的书、漫画、杂志、报 纸、唱片、绘画、盒子、邮票、明信片、照片、广告、 电影海报, 甚至艾柯自己的手绘。波利菲洛这个名字 的意思是“爱许多事物”, 但也是波莉亚——他所爱的 情人。波利菲洛寻找他失落的爱, 而波莉亚逃避波利 菲洛, 就像莉拉拒绝亚姆柏一样。莉拉是亚姆柏高中 时的暗恋对象，他的“青春期火焰”，但其时莉拉已有 男友并过早逝去。亚姆柏所怀的是一种单相思, 他高 中时给莉拉写了很多但丁体的情诗, 带有“柏拉图式 爱情”的色彩 (Eco 2009: 311-314)。但 60 岁的亚姆柏 一次偶然昏迷, 陷入失忆症, 不但丢失了很多记忆片 段, 并且在他的回忆和意识里, 他始终无法看清莉拉 的脸（因为莉拉代表了“理想”的“原型”，近于柏拉图 式的“理念”)。亚姆柏也许比他自己所想的更“泛爱 众”, 因为实际上他生命中不止一个女人。除了莉拉, 还有希比拉 (Sibilla) ——他迷人的年轻助手, 美貌 与优雅并重。更重要的是, 还有他妻子宝拉 (Paola), 这个名字与波莉亚非常接近, 她一直在他身边, 帮助 他、保护他, 达 30 多年。

在《罗安娜女王的神秘火焰》中, 如同在《寻爱 绮梦》中一样, 爱若斯 (eros) 或爱欲 (the erotic) 是与博学 (the erudite) 密不可分的。在柏拉图对话 录《会饮》中, “爱若斯”或爱欲是哲学性的, 意味着 对知识的拥有、对于美的领悟。在《罗安娜女王的神 秘火焰》中, 这一爱欲的创造性脉动的力量被亚姆柏 以神秘火焰的形式感觉到了, 这股火焰在他体内燃烧, 并在他看着那些与他童年和青少年重要而有揭示意 义的记忆相关的事物时、听着相关声音和读着相关词
句时, 感觉最为强烈。爱欲与智识融合得如此密切, 亚姆柏的第二次昏迷要么归诸于他中学同学告诉他 的关于莉拉之死的消息，要么归诸于他的智识发现： 他在老家索拉腊大宅的阁楼上惊人地找到了 1623 年 的初版本莎翁全集（Eco 2009: 290）。

《寻爱绮梦》可能对艾柯的小说观也有一些启示。 在这本文艺复兴小说中, 波利菲洛的冒险之旅几乎全 发生在一个梦中。亚姆柏很经常地思考现实与梦境的 不同，小说的第三部分是在他处于昏迷之中时发生的。 亚姆柏在他昏迷而又清醒的意识中思考: 昏睡中想起 的一切记忆是否发生在自己身上? 会不会记忆中的 人都是“我”的想象和幻觉, 而“我”是另一个人? 内部 之外是不是有一个“平行世界”? 会不会只是对大脑 的刺激使然, 而实际上一切都只是“我”的梦, 而“我” 的梦是受到外界控制的（Eco 2009: 452-454）? 这实 际上是艾柯在质疑当代符号学自我的构成, 也是向读 者的发问: 个体所感知的“现实”在多大程度上依托于 人的意识, 又在多大程度上依托于个体之外的客观事 物?

亚姆柏小时候看过一本女性杂志的封面女人画 像, 那幅画像给了他一个关于女性的“完美形象”, “一 个永远达不到的理想”和“原型” (Eco 2009: 277)。这 个“原型”和他的妻子宝拉、他的初恋莉拉及他的助手 希比拉之间存在一种转喻关联, 这使他意识到他一生 都在追逐同一个女人。亚姆柏在他所爱者们( beloved) 身上看到了点燃激情这样的可能性, 同时, 这些爱也 带他趋近死亡。他的第二次昏迷就是出现在他听说了 他的初恋莉拉之死之后, 而他再也没有醒来。《罗安 娜女王的神秘火焰》中的女人们体现了对“永恒女性” 的发现, 对欲望的发现, 对那股引导亚姆柏重建记忆 的神秘火焰的发现。仔细观察, 这股神秘火焰直接连 通着 500 年前的《寻爱绮梦》, 并将继续燃烧下去, 只要人类仍有爱与梦。

\section{4. 结论}

伟大的德国艺术史家瓦尔堡（Aby Warburg, 1866-1929）曾在博士论文中提到《寻爱绮梦》, 他对 波提切利 (Sandro Botticelli, 1445-1510) 画中“舞动的 宁芙”形象的研究受惠于《寻爱绮梦》的插图。《寻爱 绮梦》帮助他理解过去的遗存是通过哪些特殊方式在 文艺复兴想象中复活的。波提切利并没有因为模仿古 代希腊艺术而平庸, 而是通过对经典的转写和升华, 让古代“再生”, 使自己的作品也擢升为杰作 (Oettinger 225-246)。因此, 如何将僵死的知识、抽象的形式与 人类恒久而弥新的激情重新熔铸成提供当代能量的 艺术作品? 瓦尔堡发现, 对于古典、前辈和先例, 伟 大艺术家不是因循重复, 而是从中发现和汲取普遍性, 并竭力成就自己的个性、异质、独特性（Warburg 268-345)。真正伟大的艺术家并不盲从和重复古典作 者, 而是运用强大的创造力和想象力, 从久远的故纸 堆中锻造最为个人化的形式语言, 将历史和当下、个 人和整体集于一身, 并将人类自由的生命能量和强烈 
情感通过词语和图像传递下去。艾柯的《罗安娜女王 的神秘火焰》熟练操演着《寻爱绮梦》里的图文跨媒 介、新柏拉图主义和“永恒女性”母题，同时又将其置 于跟后现代思想文化的论争当中，而时间和声音维度 的加入共同实现了对中古记忆术和小说创作的革新, 达成了个体记忆与集体记忆的反思性重建 (Quanzhao 45-52)。传统及其继承虽是“旧瓶”，关键是如何酿造 “新酒”。正是在此考验着艺术家的天赋、眼力、手段 和可能达至的境界。天才从来不是“无中生有”, 而是 把创新深植于传统。他既有能力借用过往的知识-艺 术模式, 又有能力对其重新加工, 在对人类最古老、 最强烈的原始情感的体悟和复现中, 在跨文化、跨门 类的图像和艺术中, 不断跨越知识和时空的疆界, 摆 荡于想象和理性、迷狂和沉思的间隙, 释放潜藏着的 生命能量与激情, 最终呼吸到重生的自由气息。当“神 秘火焰”传递到下一个掌握了“瓦尔堡方法”的艺术家 手中, 《寻爱绮梦》便获得了死后生命 (afterlife), 并 会不断再生 (renaissance)。

\section{项目基金}

本文为 2019 年度国家社科基金一般项目《文艺 复兴时期英国文学中的忧郁书写研究》 (批准号: 19BWW056）的阶段性成果之一。

\section{REFERENCES}

[1] Bookman (Ed.) (2019). Book World (1). Jiuzhou Publishing House, Beijing.

[2] Caldwell, Ian, Thomason, Dustin (2007). The Rule of Four. Trans. Ying, Zhang and Ling, Xu. People's Literature Publishing House, Beijing.

[3] Colonna, Francesco (1999). Hypnerotomachia Poliphili: The Strife of Love in a Dream. Trans. Godwin, Joscelyn. Thames \& Hudson, New York.

[4] Eco, Umberto (2014). La memoria vegetale e altri scritti di bibliofilia. Trans. Jianquan, Wang. Yilin Press, Nanjing.

[5] Eco, Umberto(2009). The Mysterious Flame of Queen Loana. Trans. Mengzhe, Yang. Crown Publishing Co., Taiwan.

[6] Mosebach, Martin (2010). The Most Beautiful Book of the Renaissance. Trans. Ning, Liu. Foreign Literature and Art, 4: 73-78.

[7] Oettinger, April (2006). Aby Warburg's Nymph and the Hypnerotomachia Poliphili: An Episode in the Afterlife of a Renaissance Romance. Explorations in Renaissance Culture, 32.2 (winter): 225-246.

[8] Pérez-Gómez, Alberto (2018). Built upon Love. Trans. Hui, Zou. The Commercial Press, Beijing.

[9] Quanzhao, Guo (2017). The Art of Memory, Image and the Novel: On Umberto Eco's The Mysterious
Flame of Queen Loana. In Xiangyun Wang (Ed.), Foreign Language and Literature Studies. Shandong University Press, Jinan. pp. 45-52.

[10] Tonnac, Jean-Philippe de (Ed.) (2010). N'espérez pas vous débarrasser des livres. Trans. Yaling, Wu. Guangxi Normal University Press, Guilin.

[11] Warburg, Aby (2010). Sandro Botticelli's Birth of Venus and Spring: An Examination of Concepts of Antiquity in the Italian Early Renaissance. Trans. Muchun, Wan. In Jingzhong, Fan and Yiqiang, Cao (Eds.), History of Art and History of Ideas. Nanjing Normal University Press, Nanjing. IX: 268-345.

[12] Wowo, Ding and Heng, Hu (Eds.) (2011). Studies of Architecture \& Culture(III). Central Compilation $\&$ Translation Press, Beijing. 